\title{
ORIGINAL
}

\section{EVALUACIÓN ECONÓMICA DE LA MONOTERAPIA CON TOCILIZUMAB FRENTE A ADALIMUMAB EN ARTRITIS REUMATOIDE}

\author{
Federico Navarro Sarabia (1), Francisco J Blanco (2), José Ma Álvaro Gracia (3), Juan A García
} Meijide (4), José Luis Poveda (5), Darío Rubio-Rodríguez (6) y Carlos Rubio-Terrés (6).

(1) Hospital Virgen Macarena. Sevilla.

(2) Complejo Hospitalario Universitario A Coruña. A Coruña.

(3) Hospital Universitario La Princesa. Madrid.

(4) Hospital Ntra. Sra de la Esperanza. Santiago de Compostela.

(5) Hospital Universitario La Fe. Valencia.

(6) Health Value. Madrid.

\section{RESUMEN}

Fundamento: Tocilizumab (TCZ) fue superior a adalimumab (ADA) en monoterapia en la reducción de los signos y síntomas de la artritis reumatoide del adulto (AR) en pacientes intolerantes o con respuesta inadecuada a metotrexato (MTX). El objetivo del estudio fue analizar el costeefectividad de TCZ vs ADA en estos pacientes.

Métodos: Evaluación económica del coste por respuesta o remisión con TCZ $v$ ADA a partir del estudio ADACTA (horizonte temporal: 24 semanas) Criterios de respuesta clínica ACR o de remisión de la enfermedad, índice DAS28. Ámbito: Sistema Nacional de Salud. Los costes incluidos (adquisición, administración y monitorización de los medicamentos en $€$ de 2012) se obtuvieron de fuentes españolas. Se efectuaron análisis de sensibilidad simples univariantes.

Resultados: Las tasas de respuesta ACR20, ACR50 y ACR70 con TCZ y ADA se obtuvieron en el $65 \%$ y $49,4 \%(p<0,01) ; 47,2 \%$ y $27,8 \%(p<0,01)$; y en el $32,5 \%$ y $17,9 \%(\mathrm{p}<0,01)$ de los pacientes, respectivamente. La remisión DAS28 se produjo en el $39,9 \%$ y $10,5 \%$, respectivamente $(p<0,0001)$ El coste por respuesta fue menor con TCZ que con ADA (ACR20: 8.105 y 11.553 $€$; ACR50: 11.162 y $20.529 €$; ACR70: 16.211 y $31.882 €$ ) respectivamente. El coste de la remisión DAS28 fue de $13.204 €$ y $54.352 €$ respectivamente. En todos los escenarios el tratamiento con TCZ tuvo mayor eficacia y menores costes que con ADA

Conclusiones: Según este análisis, en España la monoterapia con TCZ es una estrategia eficiente frente a ADA para el tratamiento de los pacientes con AR intolerantes o con respuesta inadecuada a MTX.

Palabras clave: Artritis reumatoide. Coste-efectividad. Adalimumab. Tocilizumab.

$\overline{\text { Correspondencia }}$

Carlos Rubio Terrés

HEALTH VALUE.

C/ Virgen de Aránzazu, 21. $5^{\circ} \mathrm{B}$.

28034-Madrid

Correo electrónico: crubioterres@healthvalue.org

\section{ABSTRACT}

\section{Economic Evaluation of Rheumathoid Arthritis Monotherapy with Tocilizumab and Adalimumab}

Background: Tocilizumab (TCZ) was superior to adalimumab (ADA), as monotherapy, in reducing signs and symptoms of adult rheumatoid arthritis (RA) when methotrexate (MTX) treatment is poorly tolerated or inappropriate. The aim of the study was to analyze the cost-effectiveness of TCZ vs ADA in these patients.

Methods: Economic evaluation of the cost per response or remission of TCZ vs ADA from ADACTA (time horizon: 24 weeks). Clinical response criteria ACR or disease remission criteria, DAS28. Perspective: National Health System. The costs included (acquisition, administration and monitoring of medicines; $€$ 2012) were obtained from Spanish sources. Simple univariate sensitivity analyzes were performed.

Results: ACR20, ACR50 and ACR70 response rates with TCZ and ADA were obtained in $65 \%$ and $49.4 \%(\mathrm{p}<0.01), 47.2 \%$ and $27.8 \%$ (p $<0.01)$; and $32.5 \%$ and $17.9 \%$ ( $<<0.01)$ of patients, respectively. DAS 28 remission occurred in $39.9 \%$ and $10.5 \%$, respectively ( $\mathrm{p}<0.0001$ ). The cost per response was lower with TCZ than with ADA (ACR20: $€$ 8,105 and $€$ 11,553; ACR50: $€$ 11,162 and $€$ 20,529; ACR70: $€$ 16,211 and $€$ 31,882 ) respectively. The cost of DAS28 remission was $€ 13,204$ and $€$ 54,352 , respectively. Treatment with TCZ was dominant (more effective, with lower costs $v s \mathrm{ADA}$ ) in all scenarios analyzed.

Conclusions: According to this analysis, in Spain TCZ monotherapy is an efficient strategy $v$ ADA for treating RA patients intolerant to MTX or in which there is inappropriate response.

Keywords: Rheumatoid arthritis. Cost-effectiveness. Adalimumab. Tocilizumab. 


\section{INTRODUCCIÓN}

La artritis reumatoide (AR) es una enfermedad caracterizada por la inflamación crónica de las articulaciones ${ }^{1}$ que en España afecta al 0,5\% de la población adulta $^{2}$. En la mayoría de los casos, el curso es progresivo y conduce al daño articular irreversible, lo que tiene como consecuencia el deterioro funcional, la disminución de la calidad de vida y la mortalidad prematura de los pacientes ${ }^{1}$.

El coste anual de la AR en España se ha estimado que está entre 590-1.262 millones de euros ${ }^{3}$. Un estudio realizado por la Sociedad Española de Reumatología estimó que el $56 \%$ de los costes totales corresponden a los tratamientos farmacológicos ${ }^{4}$.

El tratamiento de la AR debe dirigirse a controlar la actividad inflamatoria, evitar la progresión de la lesión estructural articular y prevenir la invalidez de los pacientes ${ }^{1}$. En los últimos años, la utilización precoz de los agentes biológicos ha permitido establecer objetivos terapéuticos concretos, como alcanzar la remisión o bien grados bajos de actividad ${ }^{1}$. El 36,9\% de los pacientes españoles con AR recibe algún agente biológico a lo largo de la enfermedad, siendo adalimumab (ADA) [un inhibidor del factor de la necrosis tumoral (TNF)] el más prescrito $(27,3 \%)^{5}$. El 19,6\% de los agentes biológicos se administra como monoterapia ${ }^{5}$.

Tolicizumab (TCZ) es el primer anticuerpo monoclonal inhibidor del receptor de la interleucina 6 (IL-6). En combinación con metotrexato (MTX) o en monoterapia en caso de intolerancia a MTX o cuando el tratamiento continuado con él es inadecuado, está indicado en el tratamiento de la AR activa de moderada a grave, en pacientes adultos con respuesta inadecuada o intolerancia a un tratamiento previo con uno o más fármacos anti- rreumáticos modificadores de la enfermedad (FAME) o con antagonistas del TNF ${ }^{6}$.

Recientemente se han publicado los resultados del ensayo clínico ADACTA ${ }^{7}$ que ha comparado la eficacia de TCZ y ADA, ambos en monoterapia, en pacientes con AR con un valor de actividad según el índice DAS28 mayor de 5,1 (el índice DAS28 es una escala de actividad de la enfermedad que utiliza el recuento de 28 articulaciones) y con intolerancia o respuesta inadecuada a MTX. De acuerdo con este estudio, TCZ fue más eficaz que ADA en la reducción de los signos y síntomas de la $\mathrm{AR}^{7}$.

El análisis del coste-efectividad de los nuevos medicamentos, en comparación con los ya disponibles, es un instrumento de especial relevancia para la toma de decisiones del Sistema Nacional de Salud $(\mathrm{SNS})^{8}$.

Dada la mayor eficacia observada de TCZ frente a $\mathrm{ADA}^{7}$ el objetivo del presente estudio fue analizar el coste-efectividad de la monoterapia de la AR con TCZ y ADA en caso de intolerancia a MTX o cuando el tratamiento continuado con MTX es inadecuado.

\section{MATERIAL Y MÉTODOS}

Se realizó una evaluación económica desde la perspectiva del Sistema Nacional de Salud, por lo que solo se incluyeron los costes directos sanitarios derivados del manejo de la enfermedad, para calcular el ratio coste-efectividad incremental y el coste incremental por respuesta o remisión de TCZ frente a ADA a partir de los resultados de eficacia del ensayo clínico $\mathrm{ADACTA}^{7}$, los cuales se resumen en la tabla 1. La efectividad de TCZ fue superior a la de ADA en las respuestas ACR20, ACR50 y ACR70 (mejoría igual o superior al $20 \%, 50 \%$ y $70 \%$ respectivamente, según los criterios fijados por el American 
Tabla 1

Resultados del ensayo clínico ADACTA ${ }^{7}$

\begin{tabular}{|l|c|c|c|}
\hline Variable & $\begin{array}{c}\text { TCZ } \\
\mathrm{n}=163\end{array}$ & $\begin{array}{c}\text { ADA } \\
\mathrm{n}=162\end{array}$ & $\mathrm{p}$ \\
\hline Características de los pacientes & 54,4 & 53,3 & - \\
\hline Edad media (años) & 7,3 & 6,3 & - \\
\hline Duración media de la AR (años) & 6,72 & 6,76 & - \\
\hline DAS28 (media) & & \\
\hline Variables de eficacia (intención de tratar, semana 24) & $-3,3$ & $-1,8$ & $<0,0001$ \\
\hline Primaria: cambio en el valor basal de DAS28 & $39,9 \%$ & $10,5 \%$ & $<0,0001$ \\
\hline$\%$ de remisión clínica (DAS28 $<2,6)$ & $65,0 \%$ & $49,4 \%$ & $<0,01$ \\
\hline Respuesta ACR20 & $47,2 \%$ & $27,8 \%$ & $<0,01$ \\
\hline Respuesta ACR50 & $32,5 \%$ & $17,9 \%$ & $<0,01$ \\
\hline Respuesta ACR70 & & & \\
\hline
\end{tabular}

ACR20: mejoría igual o superior al 20\% según los criterios fijados por el American College of Rheumathology (igual proporcionalidad para ACR50 y ACR70); ADA: adalimumab; AR: artritis reumatoide; índice DAS28: escala de actividad de la enfermedad que utiliza el recuento de 28 articulaciones; TCZ: tocilizumab.

College of Rheumathology) ${ }^{9}$. TZC también fue superior a ADA en la tasa de remisión clínica, según el índice DAS28 de actividad de la enfermedad $(\mathrm{DAS} 28<2,6)^{10}$.

El ratio coste-efectividad incremental se calculó mediante la fórmula siguiente:

Costes con TCZ - Costes con ADA

Efectividad con TCZ-Efectividad con ADA

El análisis se hizo para un horizonte temporal de 24 semanas (la duración del estudio ADACTA).

Se analizaron los costes de adquisición de los fármacos (caso base) y los correspondientes a la administración y monitorización de los medicamentos en el análisis de sensibilidad. Todos los costes se obtuvieron de fuentes españolas habitualmente utilizadas en las evaluaciones económicas realizadas en nuestro país y se presentan en euros (€) de 2012 (tabla 2). No se analizaron los costes por reacciones adversas debido a que en el estudio ADACTA no se observaron diferencias estadísticamente significativas o clínicamente relevantes a ese respecto ${ }^{7}$.

El coste de adquisición de los tratamientos se calculó para las pautas posológicas utilizadas en el estudio ADACTA (TCZ: $8 \mathrm{mg} / \mathrm{kg}$ IV cada 4 semanas; ADA:40 mg SC cada 2 semanas, ambos en monoterapia ${ }^{7,11}$ y considerando, por tanto, que en el período de 24 semanas se administrarían 6 dosis de TCZ y 12 dosis de ADA. La dosis de TCZ (544,0 mg por paciente) se calculó para un peso corporal medio de $68 \mathrm{~kg}$, según el estudio PRA$\mathrm{XIS}^{12}$. El coste por mg de TCZ $(1,61 €)$ se estimó para las dosis de $80 \mathrm{mg}$ y $200 \mathrm{mg}$ de TCZ, por lo que el coste por dosis fue de 878,09€ (tabla 2). Para calcular el coste de adquisición de los medicamentos se aplicó el PVL con la reducción del 7,5\% establecida en el Real Decreto-Ley $8 / 2010^{13}$. Se efectuaron dos tipos de análisis respecto al coste de adquisición de los fármacos: para el coste por mg (caso base) 
Tabla 2

Costes sanitarios considerados en el estudio (€ de agosto de 2012)

\begin{tabular}{|c|c|c|}
\hline Ítem & Coste $(€)$ & Referencia \\
\hline \multicolumn{3}{|l|}{ Adquisición de los medicamentos (PVL) } \\
\hline Adalimumab (1 jeringa de $40 \mathrm{mg}$ ) & 475,58 & 11 \\
\hline Tocilizumab (vial de $80 \mathrm{mg}$ ) & 129,13 & 11 \\
\hline Tocilizumab (vial de $200 \mathrm{mg}$ ) & 322,83 & 11 \\
\hline \multicolumn{3}{|l|}{ Coste del tratamiento } \\
\hline \multicolumn{3}{|l|}{ Adalimumab } \\
\hline Dosis de $40 \mathrm{mg} \mathrm{SC}{ }^{*}$ & 475,58 & - \\
\hline 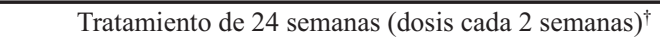 & $5.706,96$ & $\overline{-}$ \\
\hline \multicolumn{3}{|l|}{ Tocilizumab } \\
\hline Dosis de $8 \mathrm{mg} / \mathrm{kg} \mathrm{IV}$ & 878,09 & - \\
\hline Tratamiento de 24 semanas (dosis cada 4 semanas) $\|^{\|}$ & $5.268,53$ & - \\
\hline \multicolumn{3}{|l|}{ Coste de la administración** } \\
\hline Adalimumab $^{\S}$ & 1,87 & 12 \\
\hline Tolicizumab $^{\S}$ & 56,45 & 12 \\
\hline \multicolumn{3}{|l|}{ Coste de la monitorización $* *$} \\
\hline Adalimumab y Tolicizumab & 301,71 & 12 \\
\hline
\end{tabular}

IV: vía de administración intravenosa; SC: vía de administración subcutánea; PVL: precio de venta del laboratorio (se aplicó el PVL de adquisición de los medicamentos con la reducción del 7,5\% establecida en el Real Decreto-Ley $8 / 2010^{13}$ ).

** Estos costes no se consideraron en el caso base del análisis, pero sí en los análisis de sensibilidad.

* El coste de una dosis de $40 \mathrm{mg}$ de adalimumab es de 475,58€.

${ }^{\dagger}$ En el periodo de 24 semanas se administrarían 12 dosis de adalimumab.

¥ Se indica el valor adoptado en el caso base del análisis. Para un peso corporal medio de $68 \mathrm{~kg}^{12} \mathrm{se}$ administraría una dosis de 544,0 $\mathrm{mg}$ por paciente; el coste por $\mathrm{mg}$ de tocilizumab se estima en $1,61 €$ $([129,13 €+322,83 €] /[80 \mathrm{mg}+200 \mathrm{mg}])$; en consecuencia, el coste por dosis es de 878,09€ (544 mg x 1,61414€). Si el cálculo se hiciese por número de viales, el coste por dosis sería de 903,90€.

$\|$ Se indica el valor adoptado en el caso base del análisis. En el periodo de 24 semanas se administrarían 6 dosis de tocilizumab. Si el cálculo se hiciese por número de viales, el coste sería de 5.423,52€.

$\S$ Véase el texto.

๑ Se asumió que los costes semestrales de la monitorización del tratamiento (consultas y pruebas) son similares para

y para el coste por viales completos, considerando el coste de las cantidades de fármaco desechadas (análisis de sensibilidad).

De acuerdo con el estudio PRAXIS ${ }^{12}$, el coste de la administración de ADA se calculó asumiendo que el 15,6\% de las inyecciones SC las realiza una enfermera de atención primaria, para lo que dedican 4,32 minutos por inyección. En cuanto al coste de la administración de TCZ se asumió que se administra mediante perfusión IV durante 1 hora en el hospital de día ${ }^{12}$ de acuerdo con su ficha técnica ${ }^{6}$. El coste de su administración durante 1 hora en el hospital de día se estimó a partir del uso de recursos de la administración de infliximab obtenido en el estudio PRAXIS ${ }^{12} y$ considerando que: (i) El coste semestral de la administración de infliximab es de 
$735,93 €$ (actualizado a 2012); (ii) Que su frecuencia de administración es cada 7,61 semanas; (iii) Que en un semestre el número de administraciones sería de 3,42 (26 semanas/7,61 semanas); (iv) Que, en consecuencia, el coste de una administración de infliximab ascendería a 215,40 € (735,43 €/3,42 administraciones); (v) Según la ficha técnica de infliximab, éste debe ser administrado por vía intravenosa durante un período de 2 horas y los pacientes se mantendrán en observación durante al menos 1-2 horas después, debido a las reacciones agudas relacionadas con la perfusión. Por tanto, cada vial consume 3,82 horas en su reconstitución, a dministración y vigilancia $[(6,36+120,82+101,78$ minutos $) / 60$ minutos por hora); y consiguientemente (vi) el coste de 1 hora de perfusión intravenosa se calculó en 56,45 € $(215,40$ $€ / 3,82$ horas; la diferencia de 7 céntimos $(54,45-54,38)$ se debe a la contabilización de todos los decimales de los valores] ${ }^{12}$ (tabla 2).

Respecto al coste de la monitorización o seguimiento de los tratamientos con TCZ y ADA, se consideró que sería similar con ambos fármacos. El coste de las consultas y de las pruebas se obtuvo del estudio PRAXIS ${ }^{12}(301,71 €)$ (tabla 2$)$.

Con objeto de comprobar la solidez del cálculo, se realizaron análisis de sensibilidad simples univariantes en aquellos parámetros que presentaron un mayor grado de incertidumbre: (i) el coste de TCZ ajustado por el número de viales (no por $\mathrm{mg}$ ); (ii) incluyendo el coste de la administración de ambos fármacos; (iii) incluyendo el coste de la monitorización de ambos fármacos; (iv) incluyendo los costes de administración y monitorización; (v) considerando los pesos corporales de 67,0 y $69,1 \mathrm{~kg}$, correspondientes al IC $95 \%$ del peso medio obtenido en el estudio PRAXIS ${ }^{12}$.
Los resultados del análisis económico son aplicables a los pacientes que tengan características similares a los incluidos en el estudio ADACTA (tabla 1).

\section{RESULTADOS}

El coste por paciente tratado con TCZ y ADA ascendería a $5.269 €$ y $5.707 €$ respectivamente, obteniéndose un ahorro con TCZ de $438 €$.

En la tabla 3 se muestra que el coste por respuesta es menor con TCZ que con ADA (ACR20: 8.105€ y $11.553 €$; ACR50: $11.162 €$ y $20.529 €$; ACR70: $16.211 €$ y $31.882 €$ ) respectivamente, por lo que el coste de obtener una respuesta con TCZ frente a ADA se reduciría en $3.448 €, 9.367 €$ y $15.671 €$, respectivamente.

\section{Tabla 3}

Coste por respuesta ACR. Caso base del análisis

\begin{tabular}{|c|c|c|c|}
\hline $\begin{array}{c}\text { Respuesta } \\
\text { ACR }\end{array}$ & Tocilizumab & Adalimumab & $\begin{array}{c}\text { Ahorro con } \\
\text { tocilizumab }\end{array}$ \\
\hline ACR20 & $8.105 €$ & $11.553 €$ & $3.448 €$ \\
\hline ACR50 & $11.162 €$ & $20.529 €$ & $9.367 €$ \\
\hline ACR70 & $16.211 €$ & $31.882 €$ & $15.671 €$ \\
\hline
\end{tabular}

ACR20: mejoría igual o superior al 20\% según los criterios fijados por el American College of Rheumatho$\log y$ (igual porcentaje para ACR50 y ACR70).

El coste de cada remisión clínica (DAS28<2,6) fue de $13.204 €$ y $54.352 €$ con TCZ y ADA respectivamente, por lo que se produciría un ahorro por remisión de $41.148 €$ con TCZ (figura 1).

En el caso base del análisis, con un horizonte temporal de 24 semanas, el tratamiento con TCZ fue dominante (más eficaz y con menores costes que con ADA) tanto en respuesta ACR como en remisión de la enfermedad. En la tabla 4 


\section{Figura 1}

Coste por cada remisión clínica (DAS28 $<2,6$ ) obtenida con tocilizumab y adalimumab.

\section{Caso base del análisis}

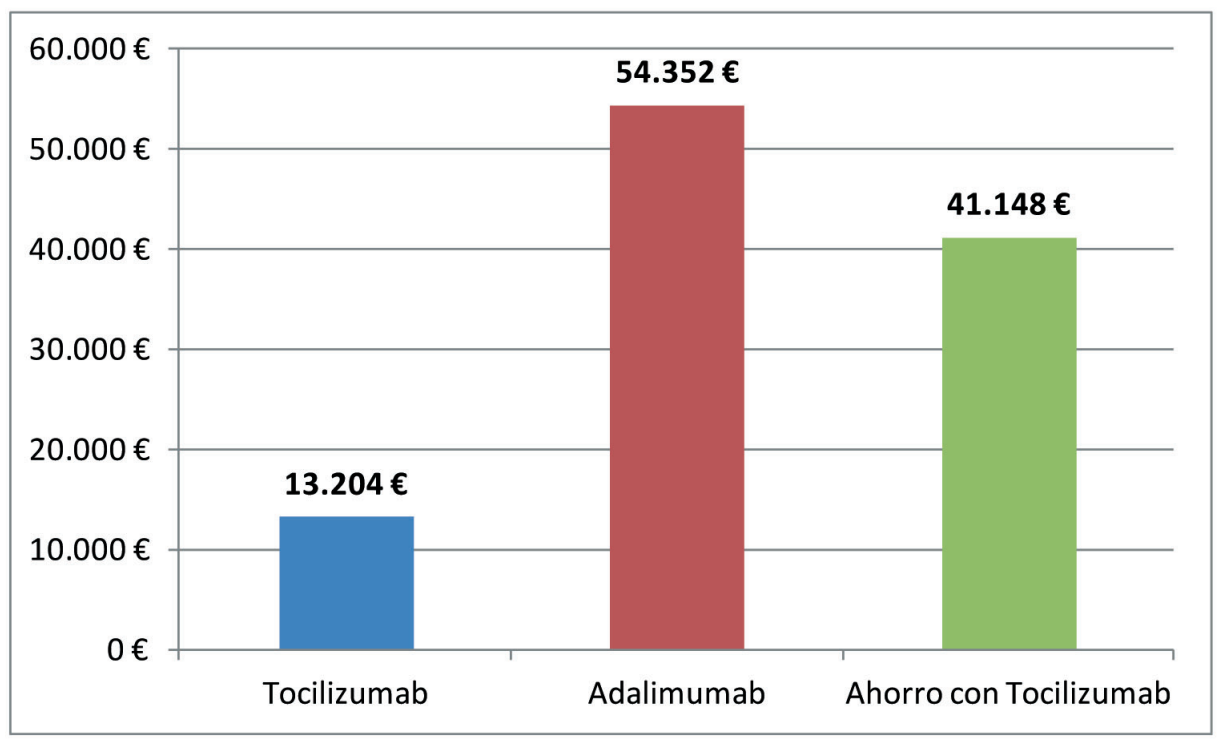

Tabla 4

Resultados de coste-efectividad: coste por remisión (DAS28 <2,6) adicional. Caso base y análisis de sensibilidad

\begin{tabular}{|c|c|c|c|c|c|c|}
\hline Análisis & Tratamiento & $\begin{array}{l}\text { Coste por } \\
\text { paciente }\end{array}$ & $\begin{array}{c}\text { Diferencia } \\
\text { de costes }\end{array}$ & $\begin{array}{c}\text { Remisión } \\
\text { DAS28 }\end{array}$ & $\begin{array}{c}\text { Diferencia } \\
\text { remisión } \\
\text { DAS28 }\end{array}$ & RCEI \\
\hline Caso base & $\begin{array}{l}\text { TCZ } \\
\text { ADA }\end{array}$ & $\begin{array}{l}5.269 € \\
5.707 €\end{array}$ & $-438 €$ & $\begin{array}{r}39,9 \% \\
10,5 \%\end{array}$ & $-29,4 \%$ & $\begin{array}{c}\text { TCZ es } \\
\text { dominante* }\end{array}$ \\
\hline \multicolumn{7}{|l|}{ Análisis de sensibilidad } \\
\hline $\begin{array}{r}\text { Coste de TCZ ajustado por el número de } \\
\text { viales }\end{array}$ & $\begin{array}{l}\text { TCZ } \\
\text { ADA }\end{array}$ & $\begin{array}{l}5.424 € \\
5.707 €\end{array}$ & $-283 €$ & $\begin{array}{l}39,9 \% \\
10,5 \%\end{array}$ & $-29,4 \%$ & $\begin{array}{c}\text { TCZ es } \\
\text { dominante* }\end{array}$ \\
\hline Se incluye el coste de administración & $\begin{array}{l}\text { TCZ } \\
\text { ADA }\end{array}$ & $\begin{array}{l}5.607 € \\
5.729 €\end{array}$ & $-122 €$ & $\begin{array}{l}39,9 \% \\
10,5 \%\end{array}$ & $-29,4 \%$ & $\begin{array}{c}\text { TCZ es } \\
\text { dominante* }\end{array}$ \\
\hline Se incluye el coste de monitorización & $\begin{array}{l}\text { TCZ } \\
\text { ADA }\end{array}$ & $\begin{array}{l}5.570 € \\
6.009 €\end{array}$ & $-438 €$ & $\begin{array}{l}39,9 \% \\
10,5 \%\end{array}$ & $-29,4 \%$ & $\begin{array}{c}\text { TCZ es } \\
\text { dominante* }\end{array}$ \\
\hline $\begin{array}{r}\text { Se incluyen los costes de administración } \\
\text { y monitorización }\end{array}$ & $\begin{array}{l}\text { TCZ } \\
\text { ADA }\end{array}$ & $\begin{array}{l}5.909 € \\
6.031 €\end{array}$ & $-122 €$ & $\begin{array}{l}39,9 \% \\
10,5 \%\end{array}$ & $-29,4 \%$ & $\begin{array}{c}\text { TCZ es } \\
\text { dominante* }\end{array}$ \\
\hline Peso corporal $=67,0 \mathrm{~kg}^{\dagger}$ & $\begin{array}{l}\text { TCZ } \\
\text { ADA }\end{array}$ & $\begin{array}{l}5.191 € \\
5.707 €\end{array}$ & $-516 €$ & $\begin{array}{l}39,9 \% \\
10,5 \%\end{array}$ & $-29,4 \%$ & $\begin{array}{c}\text { TCZ es } \\
\text { dominante* }\end{array}$ \\
\hline Peso corporal $=69,1 \mathrm{~kg}^{\dagger}$ & $\begin{array}{l}\text { TCZ } \\
\text { ADA }\end{array}$ & $\begin{array}{l}5.354 € \\
5.707 €\end{array}$ & $-353 €$ & $\begin{array}{l}39,9 \% \\
10,5 \%\end{array}$ & $-29,4 \%$ & $\begin{array}{c}\text { TCZ es } \\
\text { dominante* }\end{array}$ \\
\hline
\end{tabular}

ADA: adalimumab; índice DAS28: escala de actividad de la enfermedad que utiliza el recuento de 28 articulaciones; TCZ: tocilizumab. * TCZ domina a ADA porque es más eficaz y genera menos costes que éste. ${ }^{\dagger}$ Los pesos corporales de 67,0 y 69,1 kg corresponden al intervalo de confianza del 95\% (IC95\%) obtenido en el estudio PRAXIS ${ }^{12}$. 
se resumen los resultados obtenidos en el análisis coste-efectividad para la remisión (coste por remisión adicional obtenida con TCZ), confirmándose en todos los análisis de sensibilidad la dominancia de TCZ observada en el caso base.

\section{DISCUSIÓN}

Según el presente análisis, en España la monoterapia con TCZ es una estrategia eficiente frente a ADA para el tratamiento de los pacientes con AR que no toleran el MTX o en los que la respuesta a este fármaco es inadecuada.

El presente estudio no está exento de algunas limitaciones. En primer lugar, debe tenerse en cuenta que el coste de una hora de perfusión intravenosa de TCZ en el hospital de día es un factor que puede determinar el resultado del estudio. Ese coste se ha calculado a partir de la duración de la reconstitución, administración y vigilancia de la perfusión intravenosa de infliximab obtenida en un estudio español ${ }^{12}$ en el que se revisaron las historias clínicas de 396 pacientes con AR tratados con infliximab. Otra limitación del estudio es su breve horizonte temporal (24 semanas), acorde con la duración del ensayo clínico ADACTA en el que se basa $^{14}$. Por último, debe tenerse en cuenta que los resultados de este análisis económico son válidos para pacientes con las características de los participantes en el estudio ADACTA, ensayo clínico en el que participaron centros y pacientes españoles.

Entre las fortalezas del estudio debe mencionarse que todos los costes se obtuvieron a partir de fuentes y estudios españoles ${ }^{11,12}$ y que la totalidad de los análisis de sensibilidad realizados confirmaron la estabilidad del caso base.
No se ha identificado ningún otro análisis económico previamente publicado que analice el coste-efectividad de TCZ y ADA en monoterapia basádose en el estudio ADACTA ${ }^{7}$. Según un metaanálisis publicado por Liu et al. ${ }^{15}$ el coste por respuesta adicional ACR50 sería mayor con TCZ (31.363 \$) que con ADA (27.853 \$). Otro tanto ocurriría en ACR70 (48.320 \$ y 47.533 \$ respectivamente). Sin embargo, este estudio no incluyó el ensayo clínico ADACTA (por no estar disponible en el momento de su realización) que es el primero que ha comparado directamente la eficacia de TCZ y ADA en la AR. Por el contrario, el estudio de Liu et $\mathrm{al}^{15}$ realizó comparaciones indirectas utilizando únicamente un ensayo clínico de TCZ controlado con placebo ${ }^{16}$. A este respecto, debe considerarse que las comparaciones de eficacia (y las consiguientes evaluaciones económicas) tienen mayor validez cuando proceden de ensayos clínicos de comparaciones directas ${ }^{17}$

De acuerdo con el presente estudio, puede concluirse que en pacientes con artritis reumatoide activa de moderada a grave, con respuesta inadecuada o intolerancia a metotrexato, el tratamiento en monoterapia con tocilizumab ha demosrado ser una estrategia eficaz y rentable desde la perspectiva del Sistema Nacional de Salud en España frente al tratamiento con adalimumab.

\section{BIBLIOGRAFÍA}

1. Tornero J, Sanmartín R, Rodríguez V, Martín E, Marenco JL, González I, et al. Actualización del Documento de Consenso de la Sociedad Española de Reumatología sobre el uso de terapias biológicas en la artritis reumatoide. Reumatol Clin. 2010; 6: 23-36.

2. Carmona L, Ballina J, Gabriel R, Laffon A. The burden of musculoskeletal diseases in the general population of Spain: results from a national survey. Ann Rheum Dis. 2001;60:1040-5. 
3. Lajas C, Abásolo L, Bellajdel B, Hernández-García C, Carmona L, Vargas E, et al. Costs and predictors of costs in rheumatoid arthritis: a prevalencebased study. Arthritis Rheum. 2003; 49: 64-70.

4. Ruiz-Montesinos MD, Hernández-cruz B, ArizaAriza R, Carmona L, Ballina J, Navarro-Sarabia F, en representación del Grupo de Estudio de Costes, Calidad de vida en Artritis Reumatoide de la Sociedad Española de Reumatología. Utilización de recursos en una cohorte de pacientes con artritis reumatoide atendidos en área especializada de reumatología en España. Reumatol Clin. 2005; 1: 193-9.

5. Sociedad Española de Reumatología. Proyecto EMAR II: Variabilidad en el manejo de la artritis reumatoide y las espondiloartritis en España. Disponible en: http://www.google.com/url?sa=t\&rct=j\&q=emar\%20ii\& source $=$ web\&cd $=1 \&$ cad $=$ rja \&ved $=0$ CCEQFjAA\&url $=h t$ tp $\% 3 \mathrm{~A} \% 2 \mathrm{~F} \% 2 \mathrm{Fwww}$.ser.es $\% 2$ FArchivosDESCARGABLES\%2FProyectos\%2FEmar\%2FEMAR Informe.pdf $\&$ ei=9a9ZUJPDO8KGhQeK84DACQ\&usg=AFQjCNEC5jisJdNGDzg8hY-jadpUyfdLOg (consulta: 19 de septiembre de 2012).

6. European Medicines Agency. RoActemra. Tocilizumab. Resumen de las características del producto. RoActemra $20 \mathrm{mg} / \mathrm{ml}$ concentrado para solución para perfusión Disponible en: http://www.ema.europa.eu/ema/index.jsp?curl=page s/medicines/human/medicines/000955/human_med 001042.jsp\&mid=WC0b01ac058001d124 (consulta: 19 de septiembre de 2012).

7. Gabay C, Emery P, van Vollenhoven R, Dikranian A, Alten R, Pavelka K, Klearman M, Musselman D, Agarwal S, Green J, Kavanaugh A. ADACTA Study Investigators. Tocilizumab monotherapy versus adalimumab monotherapy for treatment of rheumatoid arthritis (ADACTA): a randomised, double-blind, controlled phase 4 trial. Lancet. 2013; 381: 1541-50.

8. Real Decreto-ley 16/2012, de 20 de abril, de medidas urgentes para garantizar la sostenibilidad del Sistema Nacional de Salud y mejorar la calidad y seguridad de sus prestaciones. BOE num 98 de 24/04/2012.

9. Arnett FC, Edworthy SM, Bloch DA, McShane DJ, Fries JF, Cooper NS et al. The American Rheumatism Association 1987 revised criteria for the classification of rheumatoid arthritis. Arthritis Rheum. 1988; 31: $315-24$.

10. Balsa A, Carmona L, Gonzalez-Alvaro I, Belmonte MA, Tena X, Sanmarti R. Value of Disease Activity Score 28 (DAS28) and DAS28-3 compared to American College of Rheumatology-defined remission in rheumatoid arthritis. J Rheumatol. 2004; $31: 40-6$
11. Base de datos de medicamentos. Consejo General de Colegios Oficiales de Farmacéuticos. (citado el 21/09/2012).Disponible en: http://www.portalfarma.com/home.nsf

12. Rubio-Terrés C, Ordovás Baines JP, Pla Poblador R, Martínez Nieto C, Sánchez Garre MJ, Rosado Souvirón MA, por el Grupo de Investigadores del Estudio PRAXIS. Utilización y coste de los modificadores biológicos de la artritis reumatoide en España (Estudio PRAXIS). Farm Hosp. 2007; 31: 78-92.

13. Real Decreto-Ley 8/2010 por el que se adoptan medidas extraordinarias para la reducción del déficit público. BOE núm 126 del 24/05/2010.

14.Gabriel S, Drummond M, Maetzel A, et al. OMERACT 6 Economics Working Group Report: A proposal for a refence case for economic evaluation in rheumatoid arthritis. J Rheumatol. 2003; 30: 886-90.

15. Liu Y, Wu EQ, Bensimon AG, Fan CP, Bao Y, Ganguli A, Yang M, Cifaldi M, Mulani P. Cost per responder associated with biologic therapies for Crohn's disease, psoriasis, and rheumatoid arthritis. Adv Ther. 2012; 29: 620-34

16. Smolen JS, Beaulieu A, Rubbert-Roth A, et al; OPTION Investigators. Effect of interleukin-6 receptor inhibition with tocilizumab in patients with rheumatoid arthritis (OPTION study): a double-blind, placebo-controlled, randomised trial. Lancet. 2008; 371: 987-97.

17. Wells GA, Sultan SA, Chen L, Khan M, Coyle D. Indirect Evidence: Indirect Treatment Comparisons in Meta-Analysis. Ottawa: Canadian Agency for Drugs and Technologies in Health; 2009. 